\title{
Automated versus manual segmentation of atherosclerotic carotid plaque volume and components in CTA: associations with cardiovascular risk factors
}

\author{
Danijela Vukadinovic • Sietske Rozie - Marjon van Gils • \\ Theo van Walsum • Rashindra Manniesing • \\ Aad van der Lugt • Wiro J. Niessen
}

Received: 31 May 2010/Accepted: 11 May 2011

(C) Springer Science+Business Media, B.V. 2011

\begin{abstract}
The purpose of this study was to validate automated atherosclerotic plaque measurements in carotid arteries from CT angiography (CTA). We present an automated method (three initialization points are required) to measure plaque components within the carotid vessel wall in CTA. Plaque components (calcifications, fibrous tissue, lipids) are determined by different ranges of Hounsfield Unit values within the vessel wall. On CTA scans of 40 symptomatic patients with atherosclerotic plaque in the carotid artery automatically segmented plaque volume, calcified, fibrous and lipid percentages were $0.97 \pm$ $0.51 \mathrm{~cm}^{3}, 10 \pm 11 \%, 63 \pm 10 \%$ and $25 \pm 5 \%$; while manual measurements by first observer were $0.95 \pm$ $0.60 \mathrm{~cm}^{3}, 14 \pm 16 \%, 63 \pm 13 \%$ and $21 \pm 9 \%$, respectively and manual measurement by second observer were $1.05 \pm 0.75 \mathrm{~cm}^{3}, 11 \pm 12 \%, 61 \pm 11 \%$ and $27 \pm 10 \%$. In 90 datasets, significant associations
\end{abstract}

D. Vukadinovic $(\bowtie) \cdot$ T. van Walsum .

R. Manniesing · W. J. Niessen

Biomedical Imaging Group, Departments of Medical Informatics and Radiology, Erasmus MC-University Medical Center Rotterdam, Rotterdam, The Netherlands e-mail: danijela.vuk@gmail.com

\section{S. Rozie - M. van Gils - A. van der Lugt}

Department of Radiology, Erasmus MC-University

Medical Center Rotterdam, Rotterdam, The Netherlands

W. J. Niessen

Faculty of Applied Sciences, Delft University

of Technology, Delft, The Netherlands were found between age, gender, hypercholesterolemia, diabetes, smoking and previous cerebrovascular disease and plaque features. For both automated and manual measurements, significant associations were found between: age and calcium and fibrous tissue percentage; gender and plaque volume and lipid percentage; diabetes and calcium, smoking and plaque volume; previous cerebrovascular disease and plaque volume. Significant associations found only by the automated method were between age and plaque volume, hypercholesterolemia and plaque volume and diabetes and fibrous tissue percentage. Significant association found only by the manual method was between previous cerebrovascular disease and percentage of fibrous tissue. Automated analysis of plaque composition in the carotid arteries is comparable with the manual analysis and has the potential to replace it.

Keywords Carotid artery - Atherosclerotic plaque · Cardiovascular risk factors - Multidetector CT angiography $\cdot$ Automated segmentation

\section{Introduction}

One of the major causes of death in the western world is atherosclerotic disease, which manifests itself as ischemic heart disease and ischemic stroke [1]. The amount of atherosclerotic disease in carotid arteries is 
normally expressed by the severity of luminal narrowing. Risk of (recurrent) stroke is related to the severity of stenosis [2]. However, the presence of a large atherosclerotic plaque is not always associated with luminal narrowing [3], which demonstrates that luminal narrowing alone is probably not a reliable marker of atherosclerosis. In addition, studies on carotid atherosclerotic plaque show that plaque morphology and composition are also important in the risk assessment of patients with carotid artery stenosis [4-6].

Atherosclerotic plaque volume and composition can be determined with Magnetic Resonance Imaging (MRI) [7-10] and CT angiography (CTA) [11-14]. CTA has established itself as an accurate modality to assess the presence of atherosclerotic disease and to grade the severity of stenosis [15]. Carotid plaques with a thin fibrous cap and a large lipid core are also considered to increase the risk for stroke [16, 17], while plaques with high calcium content, especially when located superficially, are thought to be associated with a lower risk for stroke [18].

Manual measurement of plaque volume and the contribution of the different plaque components to the plaque volume in MRI or CTA data is a very labor intensive task. Several methods address the segmentation of the outer vessel wall and plaque components in both MRI and CTA data in carotid arteries [19-24] as well as coronary arteries [25, 26]. We previously developed an algorithm to automate the plaque measurements in CTA imaging data of the carotid arteries. In this algorithm we combined outer vessel wall segmentation [27] with lumen segmentation [28]. Once the outer vessel wall and lumen were segmented, the plaque components were segmented using distinctive ranges of Hounsfield Unit (HU) values [11]. However, with this algorithm the outer vessel wall was automatically segmented only in common carotid artery, while atherosclerotic disease is commonly present in both the common and internal carotid artery.

The purpose of this study was to develop and evaluate a method to obtain automated measurements of plaque volume and its components at the carotid bifurcation and to demonstrate that this method has potential to replace the manual measurements in terms of accuracy in plaque volume and plaque component characterization. Furthermore, we investigated whether in group associations studies similar trends can be found with automated processing. Hereto, the method validation consists of (1) evaluation of the accuracy by comparing differences between method and manual tracings with variability of manual measurements of different observers and (2) comparison of the associations between cardiovascular risk factors and plaque features as assessed with manual segmentation and automated segmentations.

\section{Materials and methods}

Study population

From November 2002 to December 2005, patients with amaurosis fugax, TIA or minor ischemic stroke (Rankin score $\leq 3$ ) were consecutively enrolled in the study cohort and clinical and research data were derived in a standardized way. Multi-detector CT angiography (MDCTA) of the carotid arteries was performed as part of a research protocol, approved by the Institutional Review Board. All patients gave written informed consent. All patients underwent neurological examination on admission and symptoms and risk factors were reported. Subsequently, all carotid arteries of those patients with symptoms in the anterior circulation were evaluated for the presence of atherosclerotic plaque. This validation study of the automated plaque segmentation has a retrospective study design. The main test set contained the symptomatic carotid artery from 90 randomly selected patients $(63 \%$ male, mean age $67 \pm$ 11 years) from the group of patients with atherosclerotic plaque in the symptomatic carotid artery. The symptomatic carotid artery was the artery ipsilateral to the ischemic hemisphere, which was based on clinical symptoms and findings on MDCT of the brain. A subset of 40 datasets, which has a similar distibution of stenosis degrees as the full set, was used for the interobserver study.

\section{Training set for automated method}

The parameter settings for the automated method were previously trained on 40 manually annotated datasets, which are not part of the 90 datasets for which the method is evaluated. Furthermore this training was manually annotated by a different observer than the two observers who annotated the 
imaging data reported on this study. Hence, a possible bias of the method to one of the observers is prevented.

\section{Scan protocol and image reconstruction}

CTA of the carotid arteries was performed on a 16-slice MDCT system (Siemens, Sensation 16, Erlangen, Germany) with a standardized optimized contrast-enhanced protocol (120 kVp, $180 \mathrm{mAs}$, collimation $16 \times 0.75 \mathrm{~mm}$, table feed $12 \mathrm{~mm} /$ rotation, pitch 1) [29]. All patients received $80 \mathrm{ml}$ contrast material (Iodixanol $320 \mathrm{mg} / \mathrm{ml}$, Visipaque, Amersham Health, Little Chalfont, UK), followed by $40 \mathrm{ml}$ saline bolus chaser, each at an injection rate of $4 \mathrm{ml} / \mathrm{s}$. Synchronization between the passage of contrast material and data acquisition was achieved by real-time bolus tracking at the level of the ascending aorta. Image reconstructions were made with a 120-mm field of view, a matrix size of $512 \times 512$, a slice thickness of $1.0 \mathrm{~mm}$, an increment of $0.6 \mathrm{~mm}$, and an intermediate reconstruction filter (B46f).

Plaque volume and composition measurements

To define different plaque components by using different HU ranges, it is sufficient to have a segmentation of the carotid artery outer vessel wall and the lumen [11].

\section{Automated Segmentation of the outer vessel wall and lumen}

An automatic method using a three-point initialization was used to segment the outer vessel wall of the carotid artery in CTA [27, 30]. First, the vessel lumen was segmented using a level set approach [28], using an initialization point in the common, internal and external carotid artery. Subsequently, using a set of image features, calcium objects which are part of the vessel wall were detected using a GentleBoost framework [27]. Calcium object classification is used as a preprocessing step for the outer vessel wall segmentation since it is a much easier task than outer vessel segmentation and it can improve the accuracy of outer vessel wall segmentation. In the third step probability images were created that indicate the likeliness of a voxel lying within or outside the vessel, using the same GentleBoost framework. Each voxel is represented by a set of descriptive features: distance of the pixel to the lumen center and a set of contextual features. Contextual features in this case are radial image intensity profiles emanating from the lumen center. These profiles are extracted from the original image, the image smoothed with 2D Gaussian filters at different scales and directional 2D, Gaussian derivatives also at different scales. Based on this set of features, a GentleBoost classifier is trained to classify each pixel as being inside or outside vessel wall. The classifier provides a confidence measure which reflects the likelihood that a pixel lies inside or outside the vessel.

Finally, ellipsoids are fitted using both the calcium and vessel classification results.

\section{Manual Segmentation of the outer vessel wall and lumen}

Images of carotid arteries that contained plaque were analyzed using custom-made quantitative image analysis software, developed using MeVisLab (MeVis Research, Bremen, Germany). Using this software, regions of interest (ROI) were manually drawn over the outer vessel wall contour in consecutive axial MDCTA images (Fig. 1). Since the observers placed the ROI over the outer vessel wall contour, the ROI consisted of both plaque and lumen. The window/level setting was fixed at 1200/800 HU for all measurements.

To assess the border between lumen and atherosclerotic plaque it was necessary to draw a second ROI close to the lumen in each image. Normally, the lumen area was then automatically differentiated from atherosclerotic plaque based on the adjusted cut-off value. But in those plaques in which calcifications bordered the lumen and the two dense structures merged with each other, lumen area and calcifications had to be separated by manual drawing. One observer (S.R.) who was blinded to other clinical information, manually drew lumen and outer vessel wall contours. A second observer (M.G.) performed the manual segmentations in the interobserver dataset. A third observer (T.W.) performed manual annotation of the training set on which our automated method was trained. 

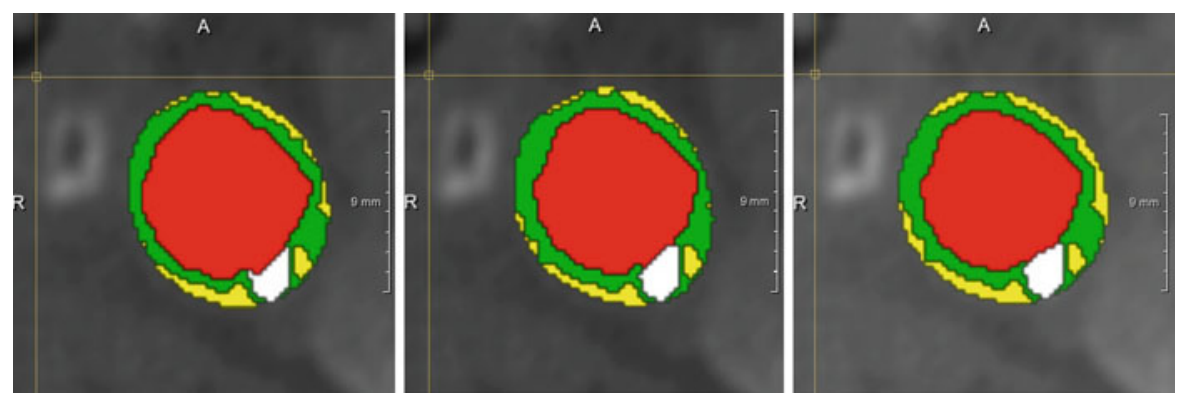

Fig. 1 Different plaque components on one cross sectional slice segmented by the automated method (left), observer S.R. (center) and observer M.G. (right). Red square lumen, Green square fibrous, yellow square lipids, white square calcium

\section{Calculation of the volume of plaque and the components}

Having the carotid artery vessel wall and lumen segmented, different $\mathrm{HU}$ ranges were used to define-different plaque components. The cut-off point for the distinction between calcifications and fibrous tissue was set at $130 \mathrm{HU}$, the value currently used for calcium scoring. The cut-off point for the distinction between fibrous tissue and lipid was set at $60 \mathrm{HU}$ as determined in previous studies [11]. We adjusted the cut-off point for the distinction between atherosclerotic plaque and vessel lumen for each patient on the basis of the full-width-half-maximum principle (mean lumen attenuation plus mean fibrous tissue attenuation $(\approx 88 \mathrm{HU})$ divided by two). The pixels surrounding the vessel lumen, with a density between $130 \mathrm{HU}$ and the adjusted cut-off value, were considered to be fibrous tissue.

The plaque volume and the volume of the plaque components were calculated by multiplying the number of pixels of the total atherosclerotic plaque or its components, with the pixel size and the slice increment. The proportion of plaque components was calculated as the ratio of volume of the component to the total plaque volume multiplied by 100 . As an example, in Fig. 1 a cross-sectional slice with outer vessel wall and all three plaque components in different colors automatically segmented and manually segmented by two observers is shown.

\section{Cardiovascular risk factors}

We obtained clinical measures and information on risk factors and medication during the patient's visit at the outpatient clinic. Subjects were categorized as currently, ever, or never smoking. Hypertension was defined as systolic blood pressure over $140 \mathrm{mmHG}$ and/or diastolic blood pressure over $90 \mathrm{mmHg}$ during two episodes of at least $15 \mathrm{~min}$ of continuous noninvasive blood-pressure measurement and/or treatment with antihypertensive medication. Blood pressure-lowering drugs comprised ACE inhibitors, calcium-antagonists, beta-blockers, and diuretics. Hypercholesterolemia was defined as fasting cholesterol over $5.0 \mathrm{mmol} / \mathrm{l}$ and/or use of cholesterollowering drugs. Diabetes was defined as fasting serum glucose levels over $7.9 \mathrm{mmol} / \mathrm{l}$, nonfasting serum glucose levels over $11.0 \mathrm{mmol} / \mathrm{l}$, or use of antidiabetic medication. Information was collected on previous cardiovascular events and conditions (myocardial infarction, atrial fibrillation, angina pectoris, chronic heart failure, coronary artery bypass grafting) and previous cerebrovascular events.

\section{Statistical analysis}

Both for automated and manual measurements ( 2 observers), plaque and plaque component volumes and proportions were presented on 40 datasets with mean $\pm(\mathrm{SD})$. The differences between automated and manual measurements and those between the measurements of two observers are presented with mean values and standard deviations. The differences between the automated and manual measurements ( 2 observers) on 40 datasets were plotted against the mean value of the measurements (Bland-Altman plot), they were evaluated with paired Student $t$ test and the correlation between the measurements by the two methods was evaluated by Pearson's correlation 
coefficient $\left(\mathrm{R}_{\mathrm{P}}\right)$. Bland-Altman plots are plotted in Excel. Limits of agreement are calculated as average difference $\pm 1.96 *$ standard deviation of the difference.

The differences between automated and manual measurements on 90 datasets (1 observer) are presented with mean values and standard deviations and the correlation was evaluated by $R_{P}$.

Both for automated and manual measurements ( 1 observer) we determined the associations between cardiovascular risk factors and PV and plaque composition on 90 datasets using univariable linear regression. Multivariable analysis was not performed for two reasons. Firstly, the focus of the study was to show that automated method can replicate the associations between manually measured plaque components and cardiovascular risk factors. Second, we did not focus on the assessment of independent associations between risk factors and plaque

Table 1 Demographic and clinical characteristics of 90 patients

\begin{tabular}{ll}
\hline Characteristics & $\mathrm{N}=90$ \\
\hline Age (years; mean $\pm \mathrm{SD}$ ) & $67 \pm 11$ \\
Male sex (\%) & 63 \\
Hypertension (\%) & 84 \\
Hypercholesterolemia (\%) & 85 \\
Diabetes mellitus (\%) & 24 \\
Smoking: current or past (\%) & 44 \\
Previous cardiac disease (\%) & 35 \\
Previous cerebrovascular disease (\%) & 31 \\
\hline
\end{tabular}

characteristics as this was not possible due to limited sample size. Because the distribution of plaque volume was skewed, we used a $\log 10$ transformation prior to statistical analysis. Similarly, for proportion of plaque components we used square root (sqrt) transformation.

$P$ values $<0.05$ were considered statistically significant. Statistical analyses were performed using SPSS software (version 15.0, Inc., Chicago, Illinois).

\section{Results}

\section{Baseline clinical characteristics}

The baseline characteristics are presented in Table 1 .

Plaque measurements: comparison to interobserver variability

Table 2 shows lumen, plaque volume, plaque component volumes and plaque component proportions assessed by the automated method and by the manual methods (two observers) in 40 datasets. The differences between the automated and the manual method and between the manual measurements of two observers are also shown. The differences between automated method and both observers were in the same range as the differences between observers:

The difference between the automated method and first observer manual measurements of PV, calcified, fibrous and lipid percentages were $0.02 \pm 0.24 \mathrm{~cm}^{3}$, $-4 \pm 6 \%,-0 \pm 8 \%$ and $4 \pm 7 \%$, respectively. The

Table 2 Plaque features measured by automated method and by two observers in 40 datasets with the differences and coefficients of variation

\begin{tabular}{|c|c|c|c|c|c|c|}
\hline 40 Datasets & $\mu(\mathrm{A}) \pm \mathrm{SD}$ & $\begin{array}{l}\mu \\
(\text { Obs } 1) \pm \mathrm{SD}\end{array}$ & $\begin{array}{l}\mu \\
(\mathrm{Obs} 2) \pm \mathrm{SD}\end{array}$ & $\begin{array}{l}\mu(\text { Diff } \\
(\mathrm{A}, \mathrm{Obs} 1)) \pm \mathrm{SD}\end{array}$ & $\begin{array}{l}\mu(\text { Diff } \\
(\mathrm{A}, \mathrm{Obs} 2)) \pm \mathrm{SD}\end{array}$ & $\begin{array}{l}\mu(\text { Diff (Obs1, } \\
\text { obs } 2)) \pm \mathrm{SD}\end{array}$ \\
\hline Plaque volume $\left(\mathrm{mm}^{3}\right)$ & $965 \pm 511$ & $946 \pm 595$ & $1052 \pm 754$ & $19 \pm 235$ & $-87 \pm 432$ & $-106 \pm 288^{*}$ \\
\hline Lumen volume $\left(\mathrm{mm}^{3}\right)$ & $1030 \pm 577$ & $1040 \pm 562$ & $1052 \pm 567$ & $-10 \pm 84$ & $-22 \pm 73$ & $-12 \pm 27^{*}$ \\
\hline Calcium volume $\left(\mathrm{mm}^{3}\right)$ & $129 \pm 178$ & $155 \pm 197$ & $136 \pm 174$ & $-26 \pm 50^{*}$ & $-6 \pm 45$ & $19 \pm 31^{*}$ \\
\hline Fibrous volume $\left(\mathrm{mm}^{3}\right)$ & $591 \pm 271$ & $564 \pm 302$ & $602 \pm 336$ & $26 \pm 101$ & $-10 \pm 165$ & $-37 \pm 112 *$ \\
\hline Lipid volume $\left(\mathrm{mm}^{3}\right)$ & $244 \pm 144$ & $225 \pm 215$ & $314 \pm 348$ & $18 \pm 133$ & $-69 \pm 272$ & $-88 \pm 190^{*}$ \\
\hline Calcium proportion (\%) & $10 \pm 11$ & $14 \pm 16$ & $11 \pm 12$ & $-4 \pm 6^{*}$ & $-1 \pm 4$ & $3 \pm 5^{*}$ \\
\hline Fibrous proportion (\%) & $63 \pm 10$ & $63 \pm 13$ & $61 \pm 11$ & $-0 \pm 8$ & $2 \pm 8$ & $2 \pm 6^{*}$ \\
\hline Lipid proportion (\%) & $25 \pm 5$ & $21 \pm 9$ & $27 \pm 10$ & $4 \pm 7 *$ & $-1 \pm 9$ & $-5 \pm 7 *$ \\
\hline
\end{tabular}

$\mu$ mean, $A$ automated method, Obs 1 first observer, Obs $2 s$ observer, $S D$ standard deviation, Diff difference, CoV coefficient of variation, $* t$ test, $P$ value $<0.05$ 
differences between automated method and second observer manual measurements of PV, calcified, fibrous and lipid percentages were $-0.09 \pm 0.43 \mathrm{~cm}^{3}$, $-1 \pm 4 \%, 2 \pm 8 \%$ and $-1 \pm 9 \%$. respectively. The differences between two observers manual measurements of PV, calcified, fibrous and lipid percentages were $0.11 \pm 0.29 \mathrm{~cm}^{3}, 3 \pm 5 \%, 2 \pm 6 \%$ and $-5 \pm 7 \%$, respectively. Similar differences were found between automated plaque measurements and manual measurements performed on 90 datasets by observer S.R.

Figure 2 shows regression plots and Pearson's correlation coefficient $\left(\mathrm{R}_{\mathrm{P}}\right)$ between automated and manual measurements by both observers of plaque features on 40 datasets. For PV, calcium, fibrous and lipid contribution correlation coefficients between automated and manual measurements of observer S.R. and M.G. were: 0.92 and $0.83 ; 0.94$ and 0.94; 0.79 and 0.73 and 0.57 and 0.52 , respectively. Correlation coefficients between automated and manual measurements by observer S.R. on 90 datasets of $\mathrm{PV}$, calcium, fibrous and lipid contributions were $0.89,0.86,0.77$ and 0.55 , respectively.

From the Bland-Altman plots, in Fig. 3, it can be observed that the difference between automated and manual measurements of plaque volume increases when the volume increases. Similarly, the difference between automated and manual measurements of plaque components proportions increase with the components proportions.

Risk factors associations with plaque features: comparison between manual and automated measurements

Table 3 shows the associations between cardiovascular risk factors and automatically assessed and manually assessed plaque volume and plaque component percentages on 90 datasets. Mostly, similar associations were found with automatically and manually assessed plaque features and cardiovascular risk factors.

Older patients had significantly larger PV measured by the automated method, whereas the association with manually assessed PV was not significant $(P=0.065)$. Male patients had significantly higher plaque volume for both automated and manual plaque measurements. Patients with hypercholesterolemia had significantly lower plaque volume when measured with the automated method, and not when measured manually. Patients who were smokers and patients who had a history of cerebrovascular disease had significantly higher plaque volume for both automated and manual plaque measurements.

For both automated and manual plaque measurements older patients had a significantly higher calcium contribution and a significantly lower fibrous contribution. Male patients had a significantly higher lipid contribution for both automated and manual plaque measurements. Patients with diabetes had a significantly higher calcium contribution for both automated and manual plaque measurements and a significantly lower fibrous contribution when measured with the automated method and not when measured manually.

Patients with a history of cerebrovascular disease had a significantly lower fibrous contribution when measured manually and not when measured automatically.

\section{Discussion}

In this study we presented a method for automated plaque volume and plaque composition assessment. Furthermore, we evaluated its accuracy (i) with respect to manual tracings, and (ii) its ability to replicate associations between plaque characteristics and cardiovascular risk factors.

With respect to segmentation accuracy, we showed that the differences in estimating plaque volume and plaque components between our automated method and expert observers are in the same range as interobserver variability. The results show some bias between the observers and between observers and the method. All automated volume measurements values are larger than volumes measured by observer S.R. and smaller than the volumes measured by observer M.G. with the exception of calcium volume and fibrous contribution. All the volumes and proportion differences between two observers were statistically significant, although these differences were small. The statistically significant difference is a consequence of a persistent, albeit small, oversegmentation of most plaque components by observer M.G. compared to observer S.R. The differences between automated method and observer S.R. were significant for calcium volume and proportion and lipid 


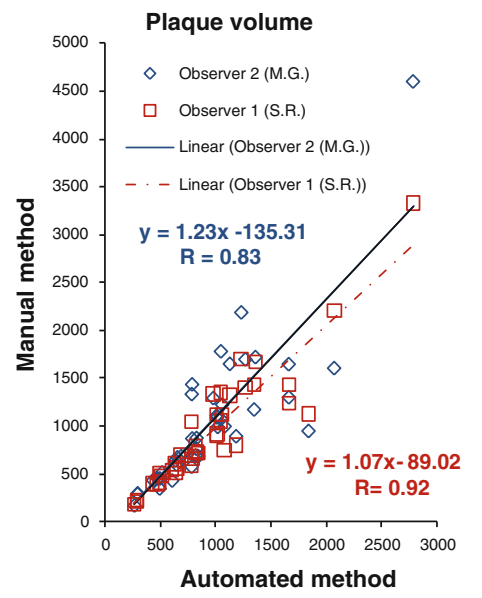

Calcium volume

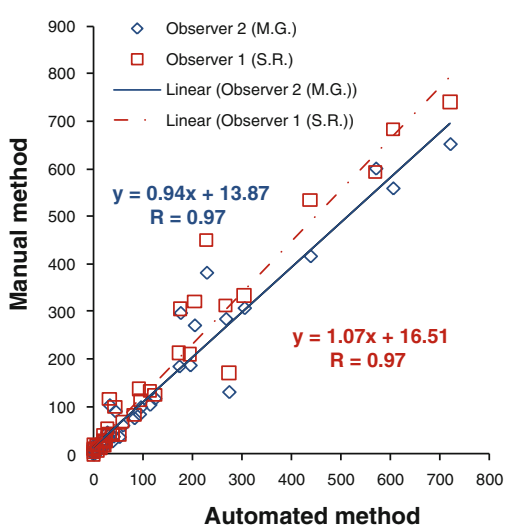

Calcium percentage

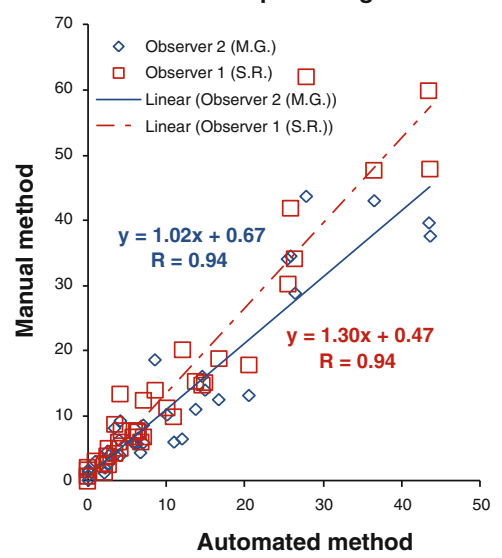

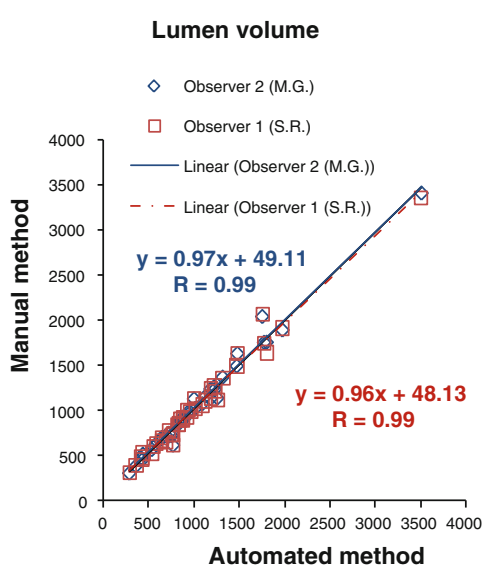
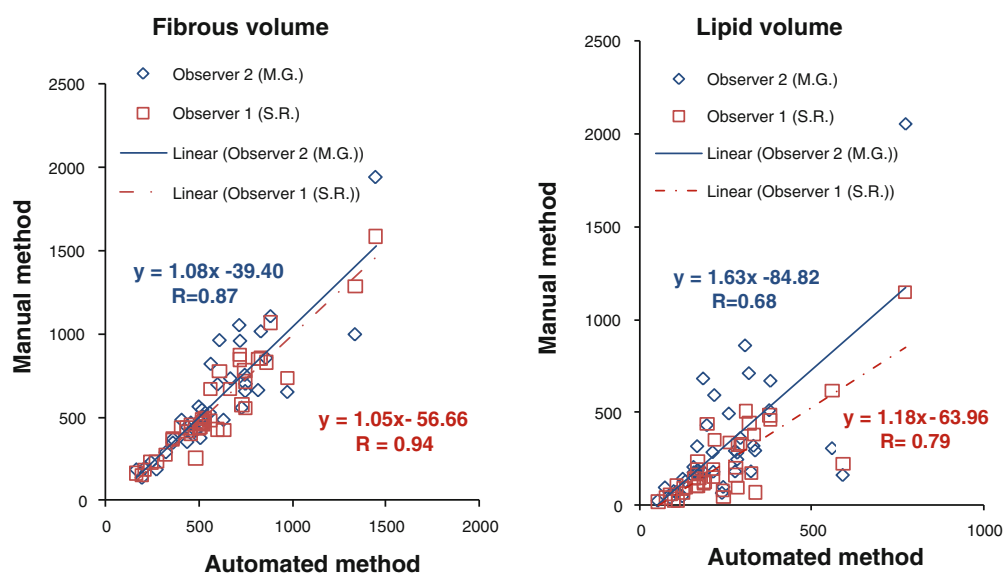

Fibrous percentage

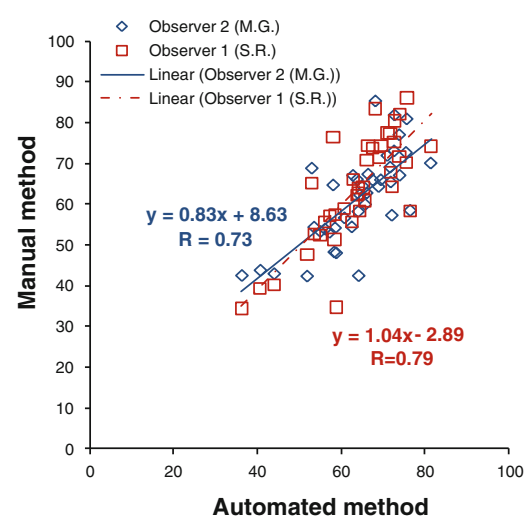

Fig. 2 Regression plots showing the comparison between automated and manual measurements in 40 datasets

proportion. None of the differences between automated method and observer M.G. were significant. The differences between automated method and observer S.R. were larger than the differences between automated method and observer M.G. for more measurements: calcium volume, fibrous volume, calcium proportion and lipid proportion. In a previous interobserver study of plaque and plaque 

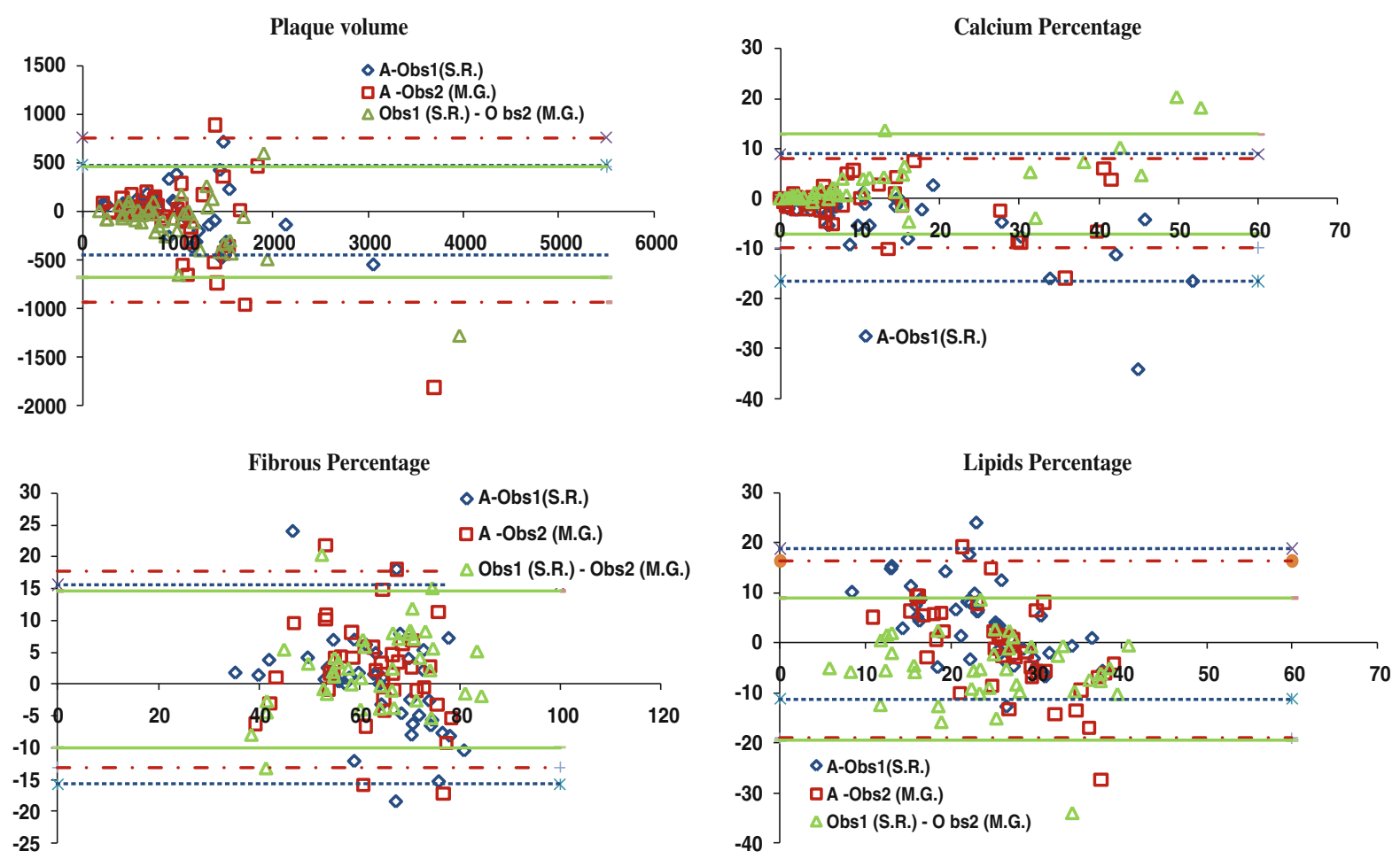

Fig. 3 Bland-Altman plots of plaque volume and plaque components percentages assessed by automated method and manual method by two observers in 40 datasets

components assessment with CTA, in which three observers manually annotated 46 CTA datasets [14], the differences of plaque volume and all plaque components were significant between at least one pair of observers [14]. Pearson's correlation coefficients between automated and manual measurements of both observers for PV and plaque contributions were mostly similar as shown in Fig. 2.

In a second evaluation, we showed that associations between cardiovascular risk factors and automated plaque measurements were mostly similar to the associations found with manual plaque measurements. Seven associations were significant for both the automated and manual plaque measurements; one was significant for the automated method and almost significant for manual method and three were significant for only one of the methods. The associations that were found to be significant for both manual and automated measurements have also similar correlation coefficient values.

To our knowledge, this is the first study that compares cardiovascular risk factors associations with manually and automatically assessed plaque volume and plaque components. When comparing our results with results of a previous study in which the association between cardiovascular risk factors and manually assessed plaque volume and plaque components was evaluated in 57 symptomatic carotid arteries [31], we found more associations. This can possible be explained by the larger number of datasets that is used in our study. In the previous study age and smoking were related to plaque volume, which is confirmed in our current study. In the previous study, patients with hypercholesterolemia had significantly less lipid and more calcium; these associations were not confirmed. A reason for this could be that automated results are the least similar to manual ones in case of lipids contribution $\left(\mathrm{R}_{\mathrm{p}}=0.55\right)$. Calcium proportion is shown to be underestimated by the automated method.

In previous studies from our institute on CT based plaque assessment [11], [29] and [14], different datasets were used than in this paper. The 57 datasets used to relate manually derived plaque measurements to cardiovascular risk factors in [31] are a subset of the 90 datasets used in this paper. Compared to this 


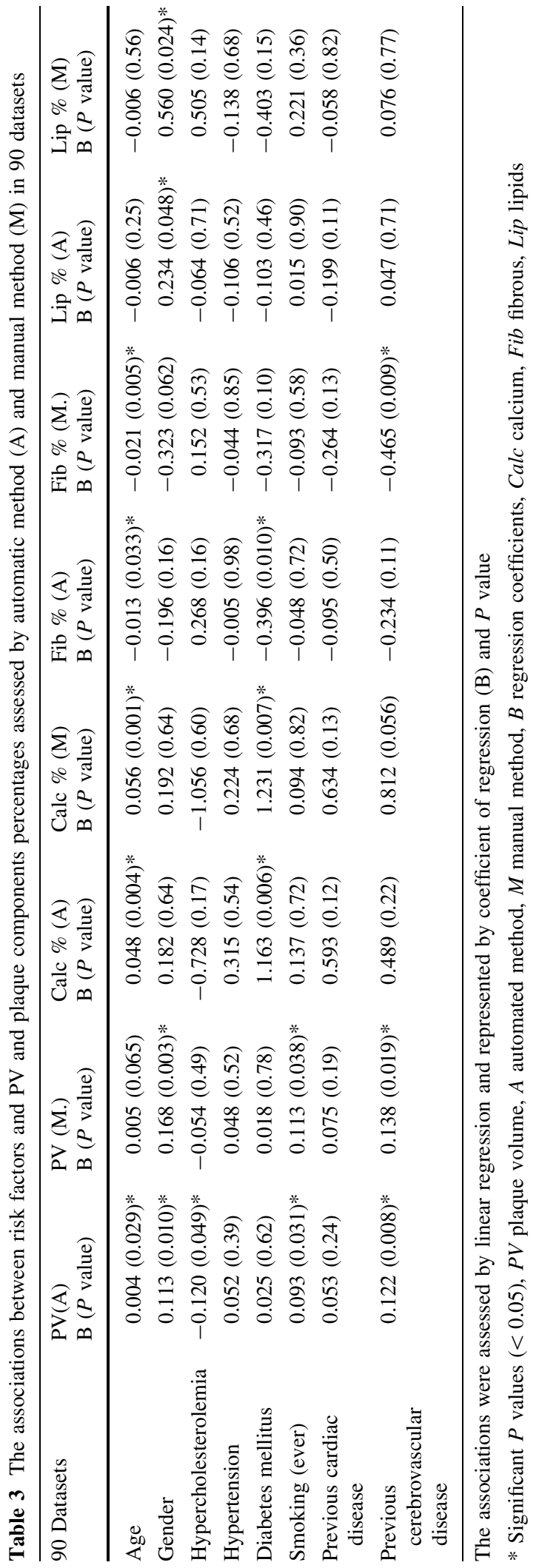

work, we thus both extended the dataset, and we investigated the influence of automated plaque assessment, in order to try to replace laborious manual plaque segmentation.

Manual quantification takes on average around $30 \mathrm{~min}$ per carotid artery. This time period depends on the lesion length. The automated method takes on average around $6 \mathrm{~min}$ on a single CPU 2GHZ, RAM 24 GB computers. Manual plaque segmentation is not applicable in clinical setting, because it is too timeconsuming. Automated plaque assessment already is considerably faster and improved implementation and hardware could reduce processing time such that it would become acceptable in clinical workflow. Also, this automated tool can be used in larger datasets for the investigation of associations between plaque volume/composition and risk factors or recurrent ischemic events and for longitudinal studies on plaque imaging.

\section{Study limitations}

A limitation of our study is that we do not have a definite gold standard. The scarcity of histological carotid plaque specimens hampers the validation of automated plaque volume and composition assessment on a sizeable dataset. We therefore compared results from automated plaque segmentation with those from manual plaque segmentation - a method previously validated against histology-thus indirectly evaluating the performance of our automated method.

A second limitation of our study is that we used CT data from a single vendor, collected at a single site. Our automated method might perform less on data from different CT-scanners, different sites or using different image protocols. However, the underlying method is generic and if required can be tuned to different systems by using new training on data. A third limitation concerns the plaque segmentation method that uses distinctive ranges of Hounsfield Unit (HU) values [11]. There is some overlap between the $\mathrm{HU}$ of lipid and fibrous tissue and no distinction can made between lipid and intraplaque heamorrhage, which is thought to be an important feature in plaque vulnerability assessment as well [9].

As a final limitation, the comparison of cardiovascular risk factors associations with manually and 
automatically segmented plaque components does only indicate that the automated method can be used to find similar associations in a group study. It does not provide information on the method applicability on a single subject.

\section{Conclusion}

We presented an approach to automatically segment outer vessel wall and plaque of carotid artery in CTA and to automatically assess plaque volume and plaque components. The results were validated with respect to manual tracings and interobserver variability. Furthermore, the associations between cardiovascular risk factors and plaque volume and plaque component contributions assessed by our automated method and a manual method were compared. We have shown that the difference between our automated method and the observers is in the range of the variability of the observers, and hence can be applied for automated analysis in large studies.

Conflict of interest None.

\section{References}

1. Robins M, Baum HM (1981) The national survey of stroke: incidence. Stroke 12:I45-I57

2. North American symptomatic carotid endarterectomy trial collaborators (1991) Beneficial effect of carotid endarterectomy in symptomatic patients with high-grade carotid stenosis. N Engl J Med 325(7): 445-453

3. Glagov S, Weisenberg E, Zarins CK, Stankunavicius R, Kolettis GJ (1987) Compensatory enlargement of human atherosclerotic coronary arteries. N Engl J Med 316(22): 1371-1375

4. Rothwell PM, Gibson R, Warlow CP (2000) Interrelation between plaque surface morphology and degree of stenosis on carotid angiograms and the risk of ischemic stroke in patients with symptomatic carotid stenosis on behalf of the European carotid surgery trialists' collaborative group. Stroke 31(3):615-621

5. Lovett JK, Gallagher PJ, Hands LJ, Walton J, Rothwell PM (2004) Histological correlates of carotid plaque surface morphology on lumen contrast imaging. Circulation 110(15):2190-2197

6. Naghavi M, Libby P, Falk E, Casscells SW, Litovsky S et al (2003) From vulnerable plaque to vulnerable patient: a call for new definitions and risk assessment strategies: part I. Circulation 108:1664-1672

7. Saam T, Hatsukami TS, Takaya N, Chu B, Underhill H, Kerwin WS, Cai J, Ferguson MS, Yuan C (2007) The vulnerable, or high-risk, atherosclerotic plaque: noninvasive MR imaging for characterization and assessment. Radiology 244:64-77

8. Cai JM, Hatsukami TS, Ferguson MS, Small R, Polissar NL, Yuan C (2002) Classification of human carotid atherosclerotic lesions with in vivo multicontrast magnetic resonance imaging. Circulation 106(11):1368-1373

9. Takaya N, Yuan C, Chu B, Saam T, Underhill H, Cai J, Tran N, Polissar NL, Isaac C, Ferguson MS, Garden GA, Cramer SC, Maravilla KR, Hashimoto B, Hatsukami TS (2006) Association between carotid plaque characteristics and subsequent ischemic cerebrovascular events: a prospective assessment with magnetic resonance imaginginitial results. Stroke 37:818-823

10. Yuan C et al (1998) Measurement of atherosclerotic carotid plaque size in vivo using high resolution magnetic resonance imaging. Circulation 98:2666-2671

11. de Weert TT, Ouhlous M, Meijering E, Zondervan PE, Hendriks JM, van Sambeek MRHM, Dippel DWJ, van der Lugt A (2006) In vivo characterization and quantification of atherosclerotic carotid plaque components with multidetector computed tomography and histopathological correlation. Arterioscler Thromb Vasc Biol 26(10):2366-2372

12. Nandalur KR, Hardie AD, Raghavan P, Schipper MJ, Baskurt E, Kramer CM (2007) Composition of the stable carotid plaque: insights from a multidetector computed tomography study of plaque volume. Stroke 38:935-940

13. Wintermark M, Jawadi SS, Rapp JH, Tihan T, Tong E, Glidden DV, Abedin S, Schaeffer S, Acevedo-Bolton G, Boudignon B, Orwoll B, Pan X, Saloner D (2008) Highresolution CT imaging of carotid artery atherosclerotic plaques. AJNR Am J Neuroradiol 29:875-882

14. de Weert T, de Monye C, Meijering E, Booij R, Niessen WJ, Dippel DWJ, Van der Lugt A (2008) Assesment of atherosclerotic carotid plaque volume with multidetector computed tomography angiography. Int $\mathrm{J}$ Cardiovasc Imaging 24:751-759

15. Koelemay MJ, Nederkoorn PJ, Reitsma JB, Majoie CB (2004) Systematic review of computed tomographic angiography for assessment of carotid artery disease. Stroke 35:2306-2312

16. Bassiouny HS, Sakaguchi Y, Mikucki SA et al (1997) Juxtalumenal location of plaque necrosis and neoformation in symptomatic carotid stenosis. J Vasc Surg 26:585-594

17. Biasi GM, Froio A, Diethrich EB et al (2004) Carotid plaque echolucency increases the risk of stroke in carotid stenting: the Imaging in Carotid Angioplasty and Risk of Stroke (ICAROS) study. Circulation 110:756-762

18. Miralles M, Merino J, Busto M et al (2006) Quantification and characterization of carotid calcium with multi-detector CT-angiography. Eur J Vasc Endovasc Surg 32:561-567

19. Yuan C, Lin E, Millard J, Hwang JN (1999) Closed contour edge detection of blood vessel lumen and outer wall boundaries in black-blood MR images. Magn Reson Imaging 17(2):257-266

20. Adams GJ, Vick GW, Bordelon CB, Insull W, Morrisett JD (2002) An algorithm for quantifying advanced carotid artery artherosclerosis in humans using MRI and active contours. Proc SPIE Medical Imaging 4684:1448-1457

21. Adame IM, van der Geest RJ, Wasserman BA, Mohamed MA, Reiber JHC, Lelieveldt BPF (2004) Automatic 
segmentation and plaque characterization in atherosclerotic carotid artery MR images. MAGMA 16(5):227-234

22. Liu F, Xu D, Ferguson MS, Chu B, Saam T, Takaya N, Hatsukami TS, Yuan C, Kerwin WS (2006) Automated in vivo segmentation of carotid plaque MRI with morphology-enhanced probability maps. Magn Reson Med 55:659-688

23. Yang F, Holzapfel G, Schulze-Bauer C, Stollberger R, Thedens D, Bolinger L, Stoplen A, Sonka M (2003) Segmentation of wall and plaque in vitro vascular MR images. Int J Cardiovasc Imaging 19:419-428

24. Kerwin W, Xu D, Liu F, Saam T, Underhill H, Takaya N, Chu B, Hatsukami T, Yuan C (2007) MRI of carotid atherosclerosis: plaque analysis. Top Magn Reson Imaging 18:371-378

25. Dey D, Cheng V, Slomka P, Nakazato R, Ramesh A, Gurudevan S, Germano G, Berman D (2009) Automated 3-dimensional quantification of noncalcified and calcified coronary plaque from coronary CT angiography. J Cardiovasc Comput Tomogr 3(6):372-382. Novet al. J Cardiovasc Comput Tomogr 2009 'Automated 3-dimensional quantification of noncalcified and calcified coronary plaque from coronary CT angiography

26. Klass O, Kleinhans S, Walker MJ, Olszewski M, Feuerlein S, Juchems M, Hoffmann MHK (2010) Coronary plaque imaging with 256-slice multidetector computed tomography: interobserver variability of volumetric lesion parameters with semiautomatic plaque analysis software. Int J Cardiovasc Imaging 26(6):711-720

27. Vukadinovic D, van Walsum T, Manniesing R, Rozie S, Hameeteman K, de Weert TT, van der Lugt A, Niessen WJ (2010) Segmentation of the outer vessel wall of the common carotid artery in CTA. IEEE Trans Med Imaging 29:65-76

28. Manniesing R, Velthuis BK, van Leeuwen MS, van der Schaaf IC, van Laar PJ, Niessen WJ (2006) Level set based cerebral vasculature segmentation and diameter quantification in CT angiography. Med Image Anal 10(2):200-214

29. de Monye C, Cademartiri F, de Weert TT, Siepman DA, Dippel DW, van der Lugt A (2005) Sixteen-detector row CT angiography of carotid arteries: comparison of different volumes of contrast material with and without a bolus chaser. Radiology 237:555-562

30. Vukadinovic D, van Walsum T, Rozie S, de Weert TT, Manniesing R, van der Lugt A, Niessen WJ (2009) Carotid artery segmentation and plaque quantification in CTA. Proceedings of IEEE international symposium on biomedical imaging, 835-838

31. Rozie S, de Weert T, de Monye C, Homburg PJ, Tanghe HLJ, Dippel DWJ, van der Lugt A (2009) Atherosclerotic plaque volume and composition in symptomatic carotid arteries assessed with multidetector CT angiography; relationship with severity of stenosis and cardiovascular risk factor. Eur Radiol 19:2294-2301 